\title{
Estudio comparado entre los sistemas de educación superior público desde el enfoque regional de movilidad y acreditación: Casos Ecuador - Argentina.
}

Comparative study between public higher education systems from the regional approach of mobility and accreditation: Ecuador - Argentina Cases.

Angela Muniz Cueva

Fecha de recepción: 09 de noviembre 2020 Fecha de aceptación: 21 de diciembre 2020 


\title{
Estudio comparado entre los sistemas de educación superior público desde el enfoque regional de movilidad y acreditación: Casos Ecuador - Argentina.
}

Comparative study between public higher education systems from the regional approach of mobility and accreditation: Ecuador - Argentina

Cases.

Angela Muniz Cueva ${ }^{1}$.

Como citar: Muniz, A. (2021). Estudio comparado entre los sistemas de educación superior público desde el enfoque regional de movilidad y

acreditación: Casos Ecuador - Argentina Revista Universidad de Guayaquil. 132(1). 33- 40. DOI: https://doi.org/10.53591/rug.v131i1.1358

\section{RESUMEN}

En el presente artículo se analizó las políticas públicas que intervienen en el sistema de educación superior con un enfoque particular en la movilidad y acreditación de la comunidad educativa de Ecuador y Argentina. En los últimos 20 años el sistema de educación superior público de Ecuador ha tenidos consistencias e inconsistencias que han impedido que el país sostenga sus acuerdos bilaterales de índole académico con Argentina u otros países de la región. Por otra parte, Argentina es un país de referente para la educación superior publica regional ya que tiene una amplia contribución académica por sus producciones literarias y científicas, esto hace que resulte atractivo para la comunidad educativa de América Latina.

Palabras clave: Sistema de educación, políticas públicas, movilidad, acreditación, cooperación

\begin{abstract}
In this article, the public politics that are included into the university educational system were analyzed with a particular approach in mobility and accreditation of the educational community in Ecuador and Argentina. In the last twenty years the public university educational system has bee having consistencies and inconsistencies, this was a hindrance for the country, because it can't keep academic bilateral agreements with Argentina. On the other hand, Argentina is a referee into the public university educational system because it has an ample academic contribution through literary and scientific production, so this country is very attractive to the educational community in Latin American.
\end{abstract}

Keywords: educational system, public politics, movility, accreditation, cooperation.

\footnotetext{
${ }^{1}$ Docente en Sociedad Beneficencia de Señoras de Guayaquil, Ecuador, Correo electrónico: munizangela321 @ gmail.com
} 


\section{INTRODUCCIÓN}

Han pasado un poco más de veinte años desde que la UNESCO público su programa de educación para todos, su enfoque a través de sus informes internacionales permite evidenciar las demandas que tanto docentes como estudiantes tienen al momento de adaptarse a las diferentes corrientes educativas, esto ha hecho un acercamiento claro a la necesidad imperiosa de que en pleno siglo XXI la demanda social por acceder a la educación superior ha ido acrecentando a nivel mundial (Ponce y Gonzáles, 2018).

Según la (UNESCO, 2020) la demanda en la matrícula superior ha incrementado en las últimas décadas y esto se evidencia en la movilidad de los estudiantes. La realidad actual es que la metamorfosis de una universidad internacional a un campus mundial de estudiantes, profesores e investigadores es irrefutable. El futuro demanda de una nueva visión y reinvención de la educación superior, este cambio debería contemplar aspectos tales como la infraestructura, conocimientos, habilidades, competencias y valores.

$\mathrm{Y}$ es precisamente que en pro de una nueva visión ante la educación superior siglo XXI se ha determinado la importancia de su dimensión internacional, la misma que requiere del intercambio de conocimiento, la creación se sistemas interactivos, la movilidad de profesores y estudiantes, así como proyectos de investigación internacionales, tomando en cuenta al menos someramente los valores culturales y las situaciones nacionales (IESALC, 2019). A partir de esta fatalidad, los países en vías de desarrollo han visto la necesidad de mejorar sus políticas de acceso a este nivel educativo. No obstante, se debe considerar que hablar de mejoras o de innovación es hablar de cambios en varias aristas que parten desde las regulaciones de normativas estatales públicas hasta la cooperación académica existente bilateralmente entre los Estados.

Planteando el enfoque específicamente en los países Latinoamericanos que quieren avanzar en este siglo XXI, resulta imperativa la regulación de las normas de acceso a la Universidad en esta Región. A modo de ejemplo, analizar los casos de Ecuador y Argentina y los cambios que han ido teniendo sus normas permitirá medir hasta qué punto está siendo efectiva la innovación adoptada por estos estados en sus Sistemas de Educación Pública Superior.

El 19 de julio del 2019 durante la Conferencia Internacional de Estados de Latinoamérica y el Caribe, representantes gubernamentales de Argentina, Bahamas, Bélice, Bolivia, Brasil, Chile, Colombia, Costa Rica, Cuba, República Dominicana, Ecuador, El Salvador, Granada, Honduras, Jamaica, México, Nicaragua, Panamá, Paraguay, Perú, Saint Kitts \& Nevis, Uruguay y Venezuela; adoptaron un nuevo convenio en el que se compromete a los Estados signatarios a adoptar todas la medidas requeridas para reconocer los estudios, títulos y diplomas de los países de América Latina y El Caribe (IESALC - UNESCO, 2019). No cabe duda que este evento representa un avance significativo para la Educación Superior Pública.

Dos aspectos importantes que el presente artículo enfocan son los aspectos de movilidad y acreditación académica. Y como el presidente boliviano Evo Morales (2017) planteó ante la Conferencia Mundial de los Pueblos "Por un mundo sin muros hacia la Ciudadanía Universal", celebrada en Tiquipaya, Bolivia; "Todos los seres humanos deben gozar de igualdad de derechos y deberes en cualquier parte del mundo". Este principio de ciudadanía universal plurinacional debe resaltar en cada una de las gestiones que emprendan los Estados en pro de la Educación Superior Pública. 
Por una parte, está Ecuador que reformó su Constitución en el 2008, garantizando una Universidad pública gratuita, sujeta al criterio de responsabilidad académica de los estudiantes; determinando que: "La gratuidad será para los y las estudiantes regulares que se matriculen en por lo menos el sesenta por ciento de todas las materias o créditos que permite su malla curricular en cada período, ciclo o nivel" (art.80 ) y "se pierde de manera definitiva la gratuidad, si un estudiante regular reprueba, en términos acumulativo, el treinta por ciento de las materias o créditos de su malla curricular cursada" (art.80).

Cabe acotar que Ecuador mantiene vigente desde el 2017 "PROYECTO DE LEY ORGÁNICA DE MOVILIDAD HUMANA", siendo entonces su eje principal en relación al presente tema de investigación el Art. 17 "Las personas ecuatorianas en el exterior podrán acceder a la educación media y superior de conformidad con las políticas implementadas por la autoridad de educación respectiva. La entidad competente facilitará el acceso a los programas de educación a distancia y virtual. En los procesos de otorgamiento de becas para realizar estudios superiores en el país y en el exterior se garantizará la participación de las personas ecuatorianas en el exterior de conformidad con la ley de la materia y sus reglamentos.

Por otra parte, el ingreso a la Universidad pública en Argentina que está reglado por la Ley 27.204 (noviembre 2015). El artículo 4 de esta ley establece que: "Todas las personas que aprueben la educación secundaria pueden ingresar de manera libre e irrestricta a la enseñanza de grado en el nivel de educación superior". Y es que de acuerdo con los expertos el sistema de Educación Superior Pública en Argentina cuenta con un sistema de acreditación y acceso que se encuentra al alcance de todos los estratos sociales.

El análisis brindado, nos permite llegar a deducir que, el problema existe y evidente es Insuficientes políticas estatales en los sistemas de educación superior pública en la región sudamericana, caso Ecuador-Argentina desde el enfoque de la teoría constructivista social en relaciones internacionales. Por tanto, el artículo se fundamentará en la construcción del conocimiento a partir de las siguientes preguntas de investigación, que se relacionan con los objetivos específicos descritos más adelante:

¿Qué políticas internacionales intervienen en los procesos educativos de nivel superior en la región sudamericana en torno a la movilidad y acreditación desde el enfoque de la teoría constructivista social?

¿Cuáles son las consistencias $\mathrm{o}$ inconsistencias; $\mathrm{o}$, convergencias $\mathbf{y}$ divergencias de los sistemas de educación superior: casos Ecuador-Argentina en torno a los acuerdos internacionales de movilidad y acreditación desde el enfoque constructivista social de la región?

¿Cuáles serían los aspectos necesarios para sugerir un Plan de mejoras al Sistema de Educación Superior del Ecuador desde la Norma Internacional y desde el enfoque constructivista social de la región?

\section{METODOLOGÍA}

Metodológicamente lo detallado contribuye a mejorar procesos de acreditación y movilidad humana, siendo importante la intervención sinérgica entre los ministerios de relaciones exteriores, mesas de trabajo en los organismos regionales que propenden a mejorar la educación superior en sus países. Proponer, establecer y sostener agendas 
bilaterales será sin duda una de las estrategias que podrían ayudar a cumplir con el objetivo de darle estabilidad a los acuerdos educativos bilaterales entre Ecuador y Argentina.

$\mathrm{Su}$ enfoque es cualitativo, por lo que no requiere de hipótesis para ser comprobadas (Sampieri, 2018), asimismo, es básica, porque contribuye a la teoría de las relaciones internacionales ajustada al constructivismo social (del Arenal, 2015), además, está guiada por la metodología propositiva (Charaja, 2010), por lo que se hace necesario el estudio de un hecho fáctico, revisando sus causas, revisando teorías en el ámbito del área del conocimiento relacionada a la variable fáctica o conocida como variable dependiente, que es donde encontramos el problema. Esta investigación se centra en establecer la existencia de insuficientes políticas estatales en los sistemas de educación superior pública, donde se considera como causas principales la limitada construcción del marco legal de la política pública, presupuesto destinado a becas y apoyo logístico para la formación académica en los postgrados. Esto permite llegar a deducir que se requiere una urgente revisión de proyectos de carreras de postgrados en el Ecuador especialmente.

Una vez, concluida la investigación es necesaria la presentación de una propuesta basada en las causas, teorías, investigaciones previas y consulta a expertos; $\mathrm{y}$, con el aporte de los constructos encontrados durante la investigación bibliográfica y documental. El resultado será también un modelo de encuesta que no requiere ser aplicada pero sí sustentada y plasmada en la matriz de operacionalización de variables o denominada Matriz de Problema de Investigación Científica - MAPIC, para las futuras investigaciones que se realicen al respecto (Charaja, 2010).

Por último, la pertinencia va acorde con el artículo 123 que determina: "El Consejo de Educación Superior aprobará el Reglamento de Régimen Académico que regule los títulos y grados académicos, el tiempo de duración, número de créditos de cada opción y demás aspectos relacionados con grados y títulos, buscando la armonización y la promoción de la movilidad estudiantil, de profesores o profesoras e investigadores o investigadoras" (Consejo de Educación Superior, 2017). Es relevante mencionar el Acuerdo André Bello, suscrito en 1970, sustituido en 1990; este documento busca integrar y fomentar estrategias educativas, científicas, tecnológicas y culturales; además de favorecer la movilidad de los estudiantes en el exterior. Entre los países miembros los cuales son: Bolivia, Chile, Cuba, España, México, Panamá, Paraguay, Perú, Venezuela, República Dominicana, Colombia, Ecuador (Ministerio de Educación del Ecuador, 2017). Evidentemente, el Estado ecuatoriano ha venido involucrándose en procesos que respaldan e impulsan la movilidad humana.

\section{RESULTADOS}

Normativa de la Educación Superior Pública Ecuatoriana. Actualmente el marco normativo a nivel de educación superior que rige en el Ecuador es la Ley Orgánica de Educación Superior (LOES), su objetivo prima en la definición de principios, brindar la garantía del derecho a la educación superior de calidad que procure la excelencia, el acceso universal, permanencia, así como la movilidad y egreso sin discriminación; el Consejo de Evaluación, Acreditación y Aseguramiento de la Calidad de la Educación Superior (CEAACES) es la entidad encargada de velar por el sistema de evaluación y/o acreditación (SITEAL, 2018). 
Las normas establecidas dentro de esta ley contemplan el art.26 de la Constitución de la República en el que se hace mención que un área primordial de la política pública y de la inversión estatal es la garantía de igualdad e inclusión social, esto como condición indispensable para el buen vivir (p. 5). El art.353 es uno de los más pertinentes al presente tema de investigación ya que este hace referencia a la necesidad de que un organismo público sea el encargado de regular la relación entre los actores participes de la comunidad educativa, además de un equipo técnico encargado de velar por el proceso de acreditación y permanencia de la calidad de las instituciones (p. 6).

Partiendo entonces desde la Constitución de la Republica es pertinente indicar que la LOES cita en su art. 5, los derechos de los estudiantes entre los cuales es pertinente destacar el derecho a la movilidad y a la garantía de ser partícipe de un proceso óptimo de acreditación y evaluación (p. 7). Sin embargo, pese a la existencia de estas normas aún existen inconsistencias en su aplicación ya que con la entrada de cada gobierno hay ligeros cambios de rumbos que según uno de los expertos consultados podría deberse incluso por estrategia política.

Normativa de la Educación Superior Pública Argentina. La normativa que rige en Argentina es la Ley de Educación 24.521 de 1999 modificada por la ley 27.204/15, esta ley tiene como objetivo la formación a nivel científico, profesional y humanista, esto contemplando también la mejoría de la calidad de vida y el respeto al medio ambiente (SITEAL, 2019). Es el Ministerio de Educación, Cultura, Ciencia y Tecnología, secretaria de Políticas Universitarias, la institución que procura la evaluación y acreditación universitaria (p. 5).

Es el art. 29 de gran relevancia ya que en este se hace referencia a las relaciones de índole educativo y científico-cultural dentro del país y con países extranjeros, además de, la tercera sección de esta ley que resulta pertinente para esta investigación ya que en el art.44 cita la necesidad de cumplir con un proceso de acreditación y evaluación que está a cargo de la Comisión Nacional, se realizan autoevaluaciones y evaluaciones externas mínimo cada seis años y con base en los resultados se generan recomendaciones pertinentes que se manejan de manera pública (UNESCO, 2018).

Según el análisis realizado se puede concluir que es la constancia y vigencia de la ley 24.521 la formula exitosa aplicada por parte del Estado Argentino ya que según SITEAL el porcentaje de jóvenes escolarizados a nivel superior en Argentina es de 32.3 siendo uno de los países con mayor rango a nivel regional, mientras que Ecuador tiene un porcentaje de 21.2; según expertos esto podría atribuirse a que el acceso a la universidad pública en Argentina brinda mayor apertura ya que cuenta con un mayor número de universidades públicas en contraste con Ecuador.

Políticas Estatales de Movilidad y Acreditación en Sudamérica. Por estos días la tendencia en lo referente a educación superior a nivel mundial es impulsar la movilidad y el intercambio académico, cabe mencionar que mayoritariamente el destino predilecto es los Estados Unidos, Canadá o países europeos (ANUIES: 2000). No obstante, es analizable el hecho de que a nivel de Latinoamérica uno de los países de mayor tendencia en movilidad académica es Argentina, según investigaciones esto podría deberse a que tiene procesos similares a países como Brasil, Chile, Colombia, México y Perú (Ortiz, 2009).

Según lo investigado se puede concluir que Argentina maneja con mayor éxito su sistema de educación ya que sostiene su ley a través de los tiempos y además ha firmado convenios de índole educativo con países de la región, esto incluye a Ecuador, sin 
embargo no es este el caso del Estado ecuatoriano que a pesar de haber firmado dicho convenio con Argentina, en la actualidad no se ha sostenido intercambio académico y no resulta educativamente atractivo para los demás países latinoamericanos, esto por su limitada sostenibilidad financiera, su inconsistencia en las normas y su carencia de un sistema educativo definido.

\section{CONCLUSIONES.}

Al analizar los sistemas de educación pública de Ecuador y Argentina se pudo evidenciar que históricamente hablando es Argentina el Estado que se mantiene como un buen referente académico a nivel regional, esto como consecuencia de sus producciones literarias y científicas a nivel mundial, y por sus acuerdos bilaterales con otros países de la región. La calidad educativa de este país y sus políticas educativas consistentes han creado expectativas por parte de los actores del área educativa superior a nivel regional.

Ciertamente Ecuador sostuvo acuerdos bilaterales con Argentina en el periodo 2007 2018 firmando convenios de intercambio académico que parecieron funcionar por un tiempo, sin embargo, actualmente esto parece haber quedado en papeles ya que no se ha logrado evidenciar mayor movilidad académica entre estas comunidades educativas en los últimos tiempos. Como ya se mencionó un factor discrepante para que la movilidad académica sea fluida ha sido la casi nula visión de internacionalización por parte de los actores de la comunidad educativa superior ecuatoriana.

Finalmente, se puede concluir que los aspectos a mejorar por parte del sistema educativo ecuatoriano son, establecer normas que deberían ser aplicadas de manera objetiva, consistente y despolitizadas, así como procurar una calidad educativa que vaya más allá de los intereses parroquiales o de acreditación. Crear una visión de internacionalización seria lo óptimo para el ámbito académico ecuatoriano ya que esto podría lograr la innovación y excelencia educativa que tanto se añora.

\section{REFERENCIAS.}

ACNUR. (2011). Colombia Situation: Fronteras (Ecuador-Colombia-Venezuela).

Araujo, S. (2017). La evaluación y la acreditación universitaria en la Argentina Sonia Araujo Doctora en Filosofía y Ciencias de la Educación. Núcleo de Estudios Educacionales y Sociales, Facultad de Ciencias Humanas. Universidad Nacional del Centro de la Provincia de Bu. Revista de Educación y Derecho, 4-6.

Buen Lozano, N. d. (1997). Instituciones de derecho del trabajo y de la seguridad social. Obtenido de UNAM: https://archivos.juridicas.unam.mx/www/bjv/libros/1/139/1.pdf

Comunidad Andina de Naciones . (2018). Integración regional participativa en la Comunidad Andina . Obtenido de Proyecto INPANDES: http://www.comunidadandina.org/StaticFiles/20181211143748MemoriaINPAN DES.pdf 
Dammert, M., Bensús, V., Sarmiento, K., \& Prieto, G. (2017). Una aproximación a las fronteras desde los mercados ilegales en el Perú. FLACSO.

Elías, C. y. (2018). Movilidad estudiantil universitaria: ¿qué factores inciden en la decisión de elegir Argentina como destino? Perfiles Educativos.

Fondo de Población de las Naciones Unidas. (2010). Migración y salud en zonas fronterizas: Colombia y el Ecuador. CEPAL.

IESALC - UNESCO. (2019). CONVENIO DE RECONOCIMIENTO DE ESTUDIOS, TÍTULOS Y DIPLOMAS DE EDUCACIÓN SUPERIOR EN AMÉRICA LATINA $Y$ EL CARIBE.

IESALC. (2019). DECLARACIÓN MUNDIAL SOBRE LA EDUCACIÓN SUPERIOR EN EL SIGLO XXI: VISIÓN y ACCIÓN. CONFERENCIA MUNDIAL SOBRE LA EDUCACIÓN SUPERIOR, (págs. 98 - 114).

Manuel Dammert-Guardia, L. D. (2020). La trata de personas en los Andes: dinámicas socio-espaciales en las fronteras de Perú. ÍCONOS Revista de Ciencias Sociales.

Ministerio de Educación del Ecuador. (s.f.). Homologación de titulos del exterior.

Monje-Álvarez, C. (2011). Metodología de la investigación cuantitativa y cualitativa Guía Didáctica. Neiva, Colombia: Universidad Surcolombiana, Facultad de Ciencias Sociales y Humanas. Programa de comunicación social y periodismo.

OPAS. (2016). Pueblos indígenas de la frontera entre Ecuador y Perú trabajarán juntos en mejorar la salud binacional. Obtenido de https://www.paho.org/hq/index.php?option=com_content\&view=article\&id=128 19:pueblos-indigenas-frontera-ecuador-peru-mejoran-saludbinacional\&Itemid $=135 \&$ lang $=$ pt

Ortiz, R. G. (2009). Perspectivas de educacion America Latina.

Plan Binacional de Desarrollo de la Región Fronteriza . (30 de noviembre de 2018). Obtenido de Acta XXII.

Plan Binacional de Desarrollo de la Región Fronteriza Ecuador-Perú. (2020). Obtenido de http://planbinacional.org.ec/wp-content/uploads/2020/04/acta-de-la-xxxiiireunion-del-directorio-del-plan-binacional-23-01-2020.pdf

Secretaría Nacional de Planificación y Desarrollo. (2017). Programa Binacional de la Región Fronteriza Ecuador-Perú . Obtenido de https://www.cancilleria.gob.ec/wp-content/uploads/2019/01/PLANBINACIONAL-DE-DESARROLLO-CAPITULO-ECUADOR.pdf

Senplades - DNP - PFP. (2014). Plan Binacional de Integración Fronteriza EcuadorColombia 2014-2022. Secretaría Nacional de Planificación .

SITEAL. (2018). Ley Organica de Educación Superior.

SITEAL. (2019). Educacion Superior. 
UNESCO. (2018). Argentina - Ley Nº 24.521. Ley de Educación.

UNESCO. (2020). Reinventando el rol y el lugar del aprendizaje . Conferencia Mundial de Educación Superior, (págs. 2-3).

Urive, S. L. (29 de agosto de 2015). Movilidad estudiantil al extranjero, objetivo de la Uteq. Ciencias $M x$.

\section{CONFLICTOS DE INTERESES}

Los autores no refieren conflictos de intereses. 